\title{
Design and Characterization of a Micro-Fabricated Graphene-Based MEMS Microphone
}

\author{
Graham S. Wood, Member, IEEE, Alberto Torin, Asaad K. Al-mashaal, Leslie S. Smith, Senior Member, IEEE, \\ Enrico Mastropaolo, Michael J. Newton, and Rebecca Cheung Senior Member, IEEE
}

\begin{abstract}
We fabricate a MEMS microphone that incorporates a graphene-based membrane that vibrates in response to acoustic forcing. We employ a novel fabrication process, where a graphene/PMMA bilayer membrane is transferred over a cavity on a separate chip before being affixed to the surface of another chip containing an electrode, resulting in the fabrication of a moveable capacitor with a membrane-to-electrode gap of $8 \mu \mathrm{m}$. The gap, which is less than half the size of other reported graphene membrane-based audio transducers, allows for the device to operate with low DC bias voltages of about $1 \mathrm{~V}$ and, when integrated with a custom-designed readout circuit, demonstrates a sensitivity to sound pressure between $0.1 \mathrm{mV} / \mathrm{Pa}$ and $10 \mathrm{mV} / \mathrm{Pa}$ across the range $100 \mathrm{~Hz}$ to $20 \mathrm{kHz}$. As well as a sensitivity that is comparable to previous work, the flat frequency response is stable when the sound pressure is varied between $70 \mathrm{~dB}_{\text {SPL }}$ and $80 \mathrm{~dB}_{\text {SPL }}$, with the sensitivity value not varying by more than $0.2 \mathrm{mV} / \mathrm{Pa}$.
\end{abstract}

Index Terms-MEMS, graphene, resonators, audio sensing.

\section{INTRODUCTION}

G RAPHENE has been the subject of an extensive body of research since its first successful synthesis over a decade ago [1], with reported work focusing on the exploitation of its unique electrical and mechanical properties, including its high Young's modulus (up to $1 \mathrm{TPa}$ ) [2] and mechanical strength (up to $130 \mathrm{GPa}$ ). In addition, graphene possesses a low mass density of $2200 \mathrm{~kg} / \mathrm{m}^{3}$, comparable to silicon, which is advantageous for creating suspended structures that can be utilized to sense pressure [3] or biological elements [4]. Additional applications of graphene include the field of photodetectors [5] and spintronics [6]. For MEMS devices, recent research that has been reported suggests the utilization of graphene in accelerometers [7] and has demonstrated a flow sensor with a graphene diaphragm [8].

For audio sensing applications such as microphones, graphene-based membranes promise several advantages over membranes fabricated from other commonly used materials, such as silicon. Graphene's ability to form a one atom thick film [9] is potentially desirable for audio applications, as

Manuscript edited April 12, 2019. This work has been supported by the Engineering and Physical Sciences Research Council (EPSRC).

G. S. Wood, A. K. Al-mashaal, E. Mastropaolo, and R. Cheung are with the School of Engineering, Institute for Integrated Micro and Nano Systems, University of Edinburgh, Edinburgh, EH9 3FF, UK (e-mail: g.s.wood@ed.ac.uk; asaad.al@ed.ac.uk; e.mastropaolo@ed.ac.uk; r.cheung@ed.ac.uk).

A. Torin and M. J. Newton are with the Acoustics and Audio Group, University of Edinburgh, Edinburgh, EH8 9DF, UK (e.mail: a.torin@ed.ac.uk; michael.newton@ed.ac.uk).

L. S. Smith is with Computing Science and Mathematics, University of Stirling, Stirling, FK9 4LA, UK (e.mail: 1.s.smith@cs.stir.ac.uk). the resulting membrane, when compared to much thicker membranes made from other materials, will exhibit a larger mechanical response to a given sound pressure. A consequence of the increased mechanical sensitivity is a reduced requirement for amplification of any electrical output, which will result in lower self-noise for the integrated microphone system.

There is a large body of research concerning MEMS microphone technology, with the trend being towards miniaturization. For use in cochlear implants and hearing aids, a smaller size is advantageous with several examples of MEMS microphone devices based on silicon membranes in the literature [10], [11]. In addition, there is research into MEMS devices that are designed to detect certain frequencies by virtue of the shape and dimension of the microstructure [12]. Graphene-based membranes have been utilized as audio sensors within a professional microphone cartridge [13] and packaged within a hearing-aid sized device $(3 \mathrm{~mm} \times$ $4 \mathrm{~mm}$ ) [14]. In the previously reported devices, the spacing between the suspended membrane and the fixed backplate has varied from $18 \mu \mathrm{m}$ up to $3 \mathrm{~mm}$, which necessitates large bias voltages, up to $200 \mathrm{~V}$, to allow for capacitive sensing.

Previously reported research has demonstrated the successful fabrication of suspended graphene structures, with a thickness up to 5 atomic layers and dimensions of the suspended area up to $5 \mu \mathrm{m}$ [15], [16]. Suspended membranes have been realized using mechanical exfoliation over trenches [15] or circular cavities [16] etched out of a layer of silicon dioxide. An additional study has demonstrated a suspended structure, fabricated for use as a nanoelectromechanical switch, created by depositing a graphene layer, using chemical vapor deposition, on top of an oxide layer that is then sacrificially etched [17].

The majority of previously reported research show graphene membranes smaller than $5 \mu \mathrm{m}$ with the deflection detected using optical methods [15], [16]. Electrical detection of the vibration of a $1.5 \mu \mathrm{m} \times 5 \mu \mathrm{m}$ graphene membrane has been demonstrated by measuring the capacitance between the membrane and the underlying substrate [17]. For use in audio applications, a larger area to thickness aspect ratio is required to yield large enough mechanical responses to sound pressure to facilitate an electrical output. The fabrication of large-scale graphene membranes represents a significant challenge, since contact between membrane and substrate must be avoided. Membranes with dimensions up to $3.5 \mathrm{~mm}$ have been fabricated and characterized [18]-[20] using laser Doppler vibrometry and interferometry, requiring the use of large and bulky equipment. If complete electro-acoustic transduction can 
be integrated with millimeter-scale graphene membranes, it could pave the way for a new generation of audio transducers.

This paper presents the design, fabrication and characterization of a MEMS microphone device incorporating a graphenebased membrane. The overall dimensions of the fabricated device are $7 \mathrm{~mm} \times 7 \mathrm{~mm} \times 0.78 \mathrm{~mm}$. A graphene/poly(methyl methacrylate) bilayer has been used for the membrane, which has a total thickness of $200 \mathrm{~nm}$, six times thinner than the $1.2 \mu \mathrm{m}$ seen in previous studies [14]. The fabricated membrane has a diameter of $3.5 \mathrm{~mm}$, which results in a very high (17500) aspect ratio of diameter to thickness, but with a smaller diameter than other work [13], [14]. Despite the ultra-large diameter to thickness aspect ratio, the fabrication method that has been used allows for the gap between the membrane and a fixed electrode to be as small as $8 \mu \mathrm{m}$. The response of the membrane to audio signals has been detected through capacitive transduction, with the vibration-induced signal amplified with a custom-made MOSFET, demonstrating the ability of the graphene-based membrane to be integrated with current CMOS technology. We have characterized the audio frequency response of the device and it has been shown to be comparable to previously reported research [11], [14], while utilizing a novel and unique fabrication method.

Section II details background theory of microphones and graphene membranes; section III outlines the design and fabrication of the graphene-based membrane microphone device; section IV outlines the experimental procedure for characterizing the device; section $\mathrm{V}$ discusses the measurement results.

\section{BACKGROUND}

\section{A. Microphones}

A microphone is a device that converts varying levels of sound pressure into an electrical signal. An example is the condenser microphone, which operates using the capacitive conduction technique with a conductive membrane, which vibrates in response to an acoustic sound pressure, acting as one plate of a capacitor. Bias voltages are applied to the membrane and the fixed bottom electrode, in order that the change in capacitance can be detected by electronic circuitry.

The performance of a microphone is defined in terms of the lowest sound pressure level (SPL) that can be detected. The lowest detectable SPL is determined by the self-noise, which is the output signal produced by a microphone in the absence of any sound pressure. For lower sound pressure levels, the signal-to-noise ratio will be lower and it will be harder to distinguish the sound signal from the self-noise.

The definition for SPL is given by

$$
\mathrm{SPL}=20 \times \log _{10}\left(\frac{\bar{p}}{\bar{p}_{0}}\right)
$$

where $\bar{p}$ is the root-mean-square (RMS) sound pressure and $\bar{p}_{0}$ is the reference RMS sound pressure of $20 \mu \mathrm{Pa}$, which is the threshold of human hearing. Therefore, an RMS sound pressure of $20 \mu \mathrm{Pa}$ is equivalent to an SPL of $0 \mathrm{~dB}_{\mathrm{SPL}}$.

Another important performance metric of a microphone is the response of the output signal as a function of the sound pressure frequency, across the range of human hearing from
$20 \mathrm{~Hz}$ to $20 \mathrm{kHz}$. A flat response across as large a frequency range as possible is desired typically by microphone users to enable accurate sound recording. However, some microphones are designed to have increased sensitivity at frequencies higher than $1 \mathrm{kHz}$, to improve the recording of talking or singing. A great deal of research and development has been undertaken by microphone manufacturers to optimize the frequency response of microphones to suit particular applications.

The trend towards miniaturization has made MEMS microphones attractive for use in mobile devices, with the current generation of devices demonstrating a lowest detectable SPL of about $30 \mathrm{~dB}_{\mathrm{SPL}}$ and a flat frequency response with a sensitivity variation of less than $1 \mathrm{~dB}$, with respect to the value at $1 \mathrm{kHz}$, across the range $200 \mathrm{~Hz}$ to $1 \mathrm{kHz}$ [21].

\section{B. Graphene-Based Membranes}

Graphene is a two-dimensional material that consists of a single layer of carbon atoms in a hexagonal lattice. It has been demonstrated previously that creating thin films of graphene down to a thickness of a single-atom is possible [9] and that, coupled with its low mass density, a suspended membrane should respond to a sound pressure with a large vibration amplitude, resulting in high sensitivity.

In order to utilize a layer of graphene as an audio transducer, it is necessary to create a bilayer structure by laminating the graphene with a supporting material, such as poly(methyl methacrylate) (PMMA), which will make the membrane less susceptible to damage in harsh operating environments. Also, using a supporting layer provides protection for the graphene layer during the process of transferring the membrane to the substrate surface and allows for the suspension of larger areas. As has been reported previously [19], graphene/PMMA bilayers have been suspended successfully over cavities with dimensions up to $3.5 \mathrm{~mm}$.

In this work, a conductive graphene layer, supported by a layer of PMMA, forms the top moveable plate of a capacitor, so that capacitive transduction can be utilized to measure the frequency and amplitude of sound pressure induced membrane vibration. Utilizing a bilayer during the fabrication process allows for a membrane to be suspended above a lower electrode with a gap of less than $10 \mu \mathrm{m}$, which minimizes the bias voltages that are needed to enable electrical readout.

\section{Capacitive Transduction}

In order to sense electrically the vibration frequency and amplitude of a MEMS structure, e.g. a membrane, capacitive transduction can be employed. The measurement mechanism is based on the moveable MEMS structure acting as one plate of a parallel-plate capacitor, with the change in distance between the structure and an underlying fixed electrode resulting in a change in the capacitance. The capacitance, $C$, between two parallel plates is given by

$$
C=\frac{\varepsilon A}{d}
$$

where $\varepsilon$ is the permittivity of the material between the plates, $A$ is the area of the parallel plates and $d$ is the gap between 
them. For an audio device with a circular membrane suspended above a fixed electrode, it can be considered to be a parallelplate capacitor, provided that the electrode is located under the center and its area is small, relative to the membrane. Therefore, when the membrane deforms in response to sound pressure, equation (2) can be used to describe the change in capacitance. The motion of the membrane with respect to the bottom electrode being an out-of-plane vibration. If electrical connections are made to each plate of the capacitor, bias voltages can be applied such that the change in capacitance will produce an output signal from an appropriately designed readout circuit.

An important consideration for capacitive sensing is the pull-in voltage, which is the membrane-electrode voltage at which the membrane comes into contact with the bottom electrode [22], [23]. The use of a PMMA supporting layer, rather than just a purely graphene membrane, should result in a lower displacement during device operation, preventing pull-in from occurring. In addition to designing the bottom electrode so that it is relatively small, bias voltage values must be set so that the membrane vibrates in the linear regime. If the electrostatic attractive force is too high, then the membrane will behave in a non-linear fashion in response to sound pressure. The gap size between the membrane and the electrode, in addition to the selected DC bias voltages, will be a trade-off between the need to detect the mechanical response to sound pressure and the prevention of pull-in.

\section{DESIGN AND FABRICATION OF DEVICE}

\section{A. Fabrication Process}

The fabrication process for the microphone device is shown in the schematic of Fig. 1 and is performed in two stages. First, the substrate is prepared from a starting material of a $75 \mathrm{~mm}$ silicon wafer with a thickness of $380 \mu \mathrm{m}$. A $77 \mathrm{~nm}$ layer of silicon dioxide has been deposited using plasma-enhanced chemical vapor deposition (PECVD) (Fig. 1a). Next, a $500 \mathrm{~nm}$ layer of aluminum has been sputtered and patterned using a lift-off process (Fig. 1c), creating the lower electrode that the graphene membrane will be suspended over. To create a cavity above the lower electrode, an $8.8 \mu \mathrm{m}$ layer of silicon dioxide has been deposited with PECVD. A $3 \mu$ m layer of photoresist has been spin-coated and patterned photolithographically. The cavity has been created by etching the oxide using a 10:9 solution of $\mathrm{NH}_{4} \mathrm{~F}: \mathrm{CH}_{3} \mathrm{COOH}$ (Fig. 1e). A further $500 \mathrm{~nm}$ layer of aluminum has been sputtered and patterned to provide electrical connections to the membrane after the transfer. In addition, vias have been etched to provide connection pads to the bottom metal electrode (Fig. 1g). Lastly, the $75 \mathrm{~mm}$ wafer is diced into $7 \mathrm{~mm} \times 7 \mathrm{~mm}$ chips, each containing a single cavity.

The second stage of the fabrication is to prepare a frame that the membrane will be suspended upon. Starting with another $75 \mathrm{~mm}$ silicon wafer with a thickness of $380 \mu \mathrm{m}$ (Fig. 1b), a $7 \mu \mathrm{m}$ layer of photoresist has been spin-coated and patterned using photolithography before having cavities etched through the wafer using deep reactive ion etching (DRIE) (Fig. 1d). The wafer has been diced into $5 \mathrm{~mm} \times 5 \mathrm{~mm}$ chips. Separately,

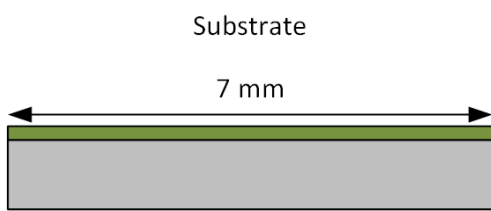

(a)

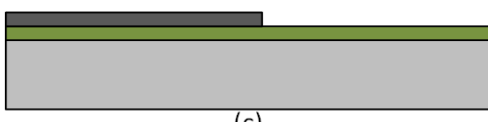

(c)



(e)

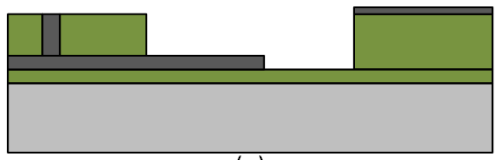

(g)
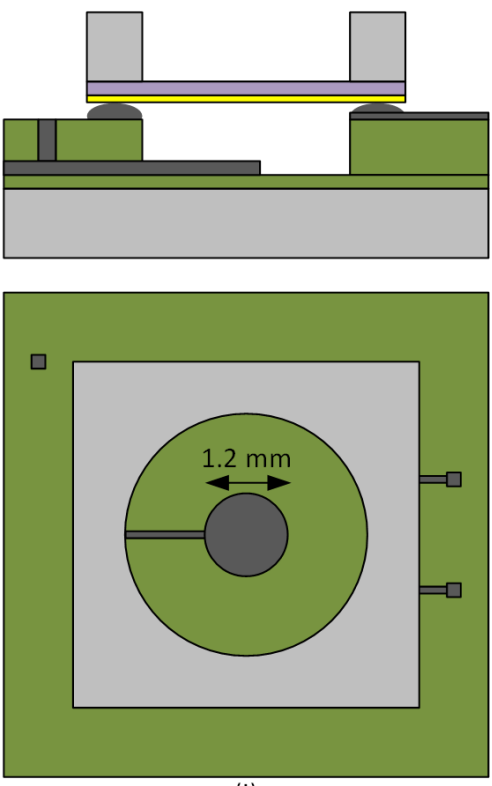

(i)

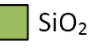

$\mathrm{Al}$

PMMA Graphene

Fig. 1. Fabrication process flow for graphene/PMMA membrane microphone device: (a) $7 \mathrm{~mm} \times 7 \mathrm{~mm}$ Si chip with $\mathrm{SiO}_{2}$ passivation layer (b) $5 \mathrm{~mm}$ $\times 5 \mathrm{~mm}$ Si chip (c) Al deposition on substrate (d) DRIE of cavity to create a frame (e) Deposition and etch of $8 \mu \mathrm{m}$ layer of $\mathrm{SiO}_{2}$ to create cavity above lower electrode (f) Transfer of graphene/PMMA membrane to frame (g) Creation of top and bottom electrode connections (h) Inversion of frame (i) Frame affixed to chip with conductive silver adhesive. Top and side view of completed device.

a sheet of membrane material has been prepared by CVD depositing a multilayer of graphene on a $200 \mathrm{~nm}$ layer of PMMA (performed by Advanced Graphene Products Sp. Z o.o.). A $5 \mathrm{~mm} \times 5 \mathrm{~mm}$ piece of the graphene/PMMA sheet has been cut out using a razor blade and carefully positioned on the frame. The frame has been coated with a small amount of deionized water to promote adhesion of the membrane. After 
the transfer has been performed (Fig. 1f), the frame is placed on a hotplate at $80{ }^{\circ} \mathrm{C}$ for 5 minutes to evaporate any DI water and at $110{ }^{\circ} \mathrm{C}$ for 10 seconds to induce an expansion of the PMMA, ensuring as flat a membrane as possible.

The complete device has been assembled by first inverting the frame with the graphene/PMMA membrane (Fig. 1h). In order to affix the frame to the substrate chip and to provide an electrical connection from the graphene layer to the bonding pads, droplets of silver adhesive have been applied to the substrate chip using a precision fluid dispensing system. The droplets have been dispensed around the cavity on the metal tracks leading to bonding pads. The frame has then been positioned on top of the substrate chip, with the cavity of the frame aligned with the cavity on the substrate chip. Pressure has been applied to ensure adhesion of the two chips and to bring the gap between the membrane and the bottom electrode down to $8.8 \mu \mathrm{m}$, confirmed with optical microscopy, as will be explained in section III-C. The completed device has overall dimensions of $7 \mathrm{~mm} \times 7 \mathrm{~mm} \times 0.78 \mathrm{~mm}$.

\section{B. Device Layout}

The top and side view of a completed device are shown in Fig. 1i. The device consists of a circular graphene/PMMA membrane with a diameter of $3.5 \mathrm{~mm}$ suspended above a circular aluminum electrode with a diameter of $1.2 \mathrm{~mm}$. The electrode has been positioned beneath the center of the membrane in order to maximize the capacitance change that will be induced for a given vibration amplitude, which will maximize the output signal. To allow for wire-bond connections to be made to the device, metal tracks have been designed that connect the membrane and the bottom electrode to $200 \mu \mathrm{m}$ $\times 200 \mu \mathrm{m}$ aluminum pads that have been positioned $400 \mu \mathrm{m}$ from the perimeter of the chip. The overall size of the chip is $7 \mathrm{~mm} \times 7 \mathrm{~mm}$, which has been designed to be comparable to other MEMS devices used in hearing aid applications.

From the fabrication process and the designed dimensions, (2) has been used to calculate the capacitance, $C$, between the membrane and the bottom electrode as follows

$$
\begin{aligned}
C & =\frac{\varepsilon A}{d} \\
& =\frac{8.85 \times 10^{-12} \times \pi \times(600 \mu \mathrm{m})^{2}}{8 \mu \mathrm{m}} \\
& =1.3 \mathrm{pF}
\end{aligned}
$$

The $1.13 \mathrm{~mm}^{2}$ area of the capacitor created by the membrane and the bottom electrode should allow for the detection of membrane deflection caused by sound pressure. The capacitance value of $1.3 \mathrm{pF}$ is larger than previously reported devices, as a consequence of the larger area and narrower gap between the membrane and the fixed electrode, allowing for smaller bias voltages to be used. For this work, a readout circuit including a custom-made MOSFET has been designed and constructed and will be detailed in section IV.

The design of the device will allow for DC bias voltages to be applied to the membrane without causing a deflection large enough to result in pull-in. Finite-element-method (FEM)

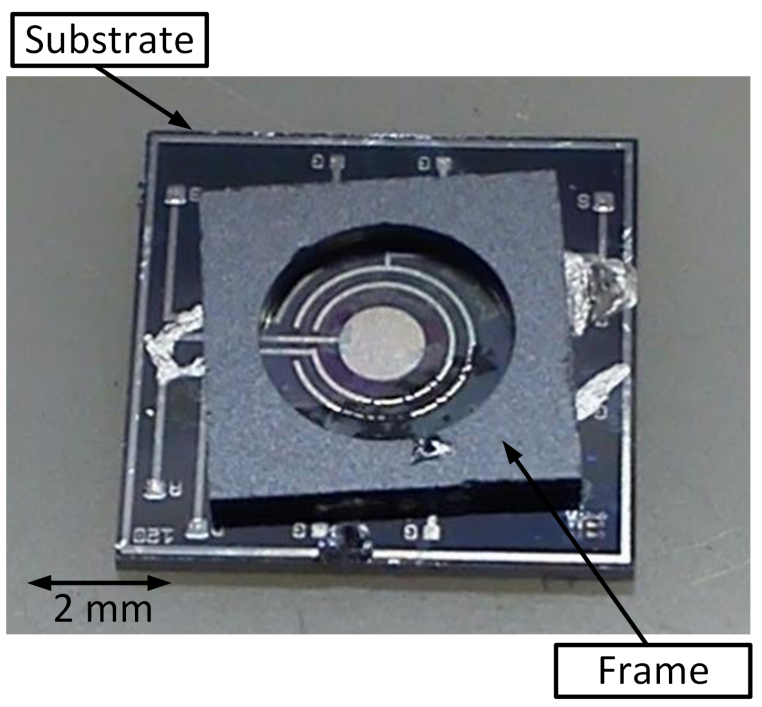

Fig. 2. Optical image of the fabricated microphone device.

simulation with CoventorWare has been performed to determine the pull-in voltage. A tension of $0.2 \mathrm{~N} / \mathrm{m}$ has been added to the membrane layer in the model, a value which has been assumed based on previously reported measurements with graphene/PMMA membranes of the same area [19]. The simulation found that pull-in occurs when the potential difference between the membrane and the underlying electrode reaches $80 \mathrm{~V}$. As will be discussed in section IV, the voltage drop between the membrane and the underlying electrode is set to $0.2 \mathrm{~V}$, so it can assumed that pull-in will not occur.

\section{Inspection of Completed Device}

An optical microscope image of a completed device is shown in Fig. 2. In addition, scanning electron microscopy (SEM) with a TESCAN VEGA3 has been used to produce the image in Fig. 3. The bottom electrode is visible in the optical image due to the transparency of the membrane to visible light, while the SEM image shows the membrane held in place by the frame.

The silver adhesive that has been used to secure the frame to the chip has been positioned such that when the frame and chip have been pressed together, no adhesive enters the cavity below the membrane. As can be seen in Fig. 2, the adhesive under the frame is also present outside of the frame on the chip, with the conductive adhesive making a connection between the membrane and the aluminum tracks that lead to bonding pads. The connection has been tested by measuring the resistance between pads on opposite sides of the chip and a conductive path through the membrane has been confirmed. The successful suspension of the membrane above the bottom electrode has been tested by ensuring electrical isolation between the pads connected to the membrane and the bottom electrode. In addition, optical microscope inspection of the device has determined that there is a gap of $8.8 \mu \mathrm{m}$ between the membrane and the bottom electrode. 


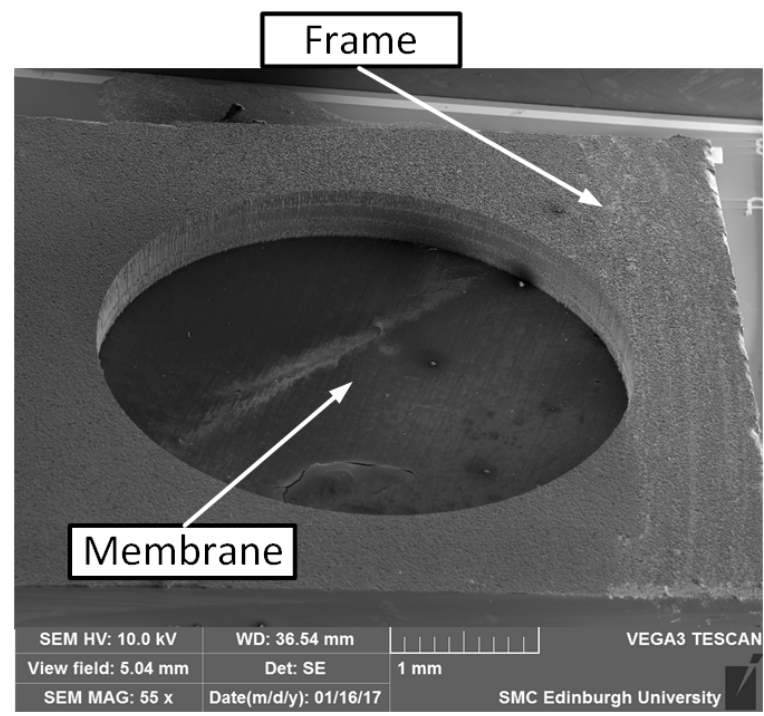

Fig. 3. SEM image of the fabricated microphone device.

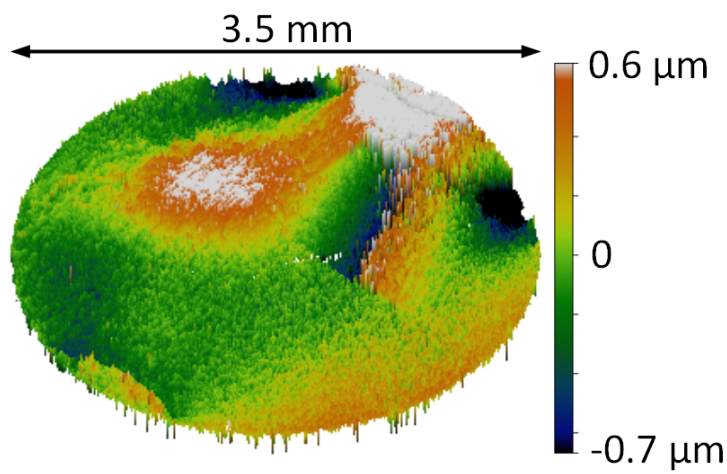

Fig. 4. Topography of membrane determined using white-light interferometry. Values are defined as the height difference from the mean.

\section{White-Light Interferometry}

In addition to the optical and scanning electron microscopy inspection of the device, white-light interferometry (Zygo) has been performed to measure the topography of the membrane to determine the success of the fabrication process. The result of a scan is shown in Fig. 4 and shows that the difference between the highest and lowest point of the membrane is $1.3 \mu \mathrm{m}$. The variation in membrane height that has been measured is most likely a result of the manual method employed to transfer the membrane to the frame. However, the small variation, relative to the membrane diameter, that has been achieved is sufficient to ensure suspension of the membrane above the underlying electrode.

From the white-light interferometry measurement data, the graph of Fig. 5 showing the profile of the membrane across the diameter has been extracted. It can be seen that, so long as the frame is at least $1.5 \mu \mathrm{m}$ above the substrate, the membrane is unlikely to come into contact with the bottom electrode. For this device, the membrane height above the bottom electrode has been already determined optically to be $8.8 \mu \mathrm{m}$, so it can be concluded that the membrane is suspended.

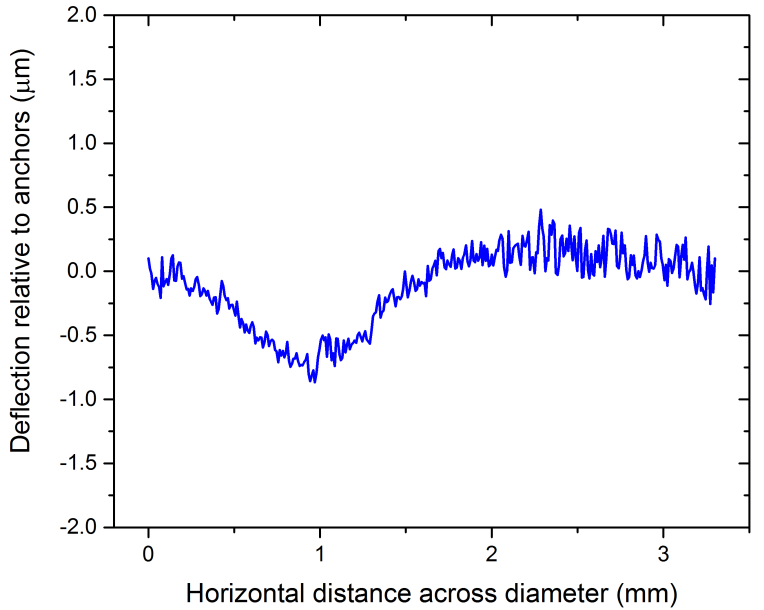

Fig. 5. Deflection of membrane across its diameter measured with white-light interferometry.

The fully characterized device has been secured in a chip carrier and wire-bonded to create electrical connections to the membrane and the bottom electrode, ready for audio testing.

\section{ExPERIMENTAL PRocedure}

After wire-bonding, the chip carrier has been secured in a socket on a printed circuit board, allowing for connections to be made to voltage sources and to readout circuitry.

The membrane and the bottom electrode form a variable capacitor, $C_{M}$, which has the static value calculated in (3). The two plates are connected to a DC voltage, $V_{C}$, and to the gate terminal of a MOSFET that forms part of the readout circuit, as shown in Fig. 6. The MOSFET has been fabricated on a separate chip and has been connected in series with a resistor, $R_{D}$, so that a voltage divider is created between the supply voltage, $V_{D D}$, and ground. The gate voltage, $V_{G}$, of the MOSFET is determined by the bias voltage, $V_{C}$, as follows [24]

$$
V_{G}=V_{C}-\frac{Q_{0}}{C_{M}}
$$

where $\mathrm{Q}_{0}$ is the total charge on the connected terminals of the MOSFET gate and the bottom electrode on the microphone chip. From (2), the capacitance, $C_{M}$, is related to the deflection, $x$, of the membrane as follows

$$
C_{M}=\frac{\varepsilon A}{d-x}
$$

where $d$ is the initial gap between the membrane and the bottom electrode. If (5) is substituted into (4), $V_{G}$ can be expressed as

$$
V_{G}=V_{C}-\frac{Q_{0} d}{\varepsilon A}+\frac{Q_{0} x}{\varepsilon A}
$$

If a sound pressure at a constant frequency, $\omega_{S P}$, is directed at the membrane, the deflection, $x$, as a function of time is given by

$$
x=x_{\text {peak }} \sin \left(\omega_{S P} t\right)
$$




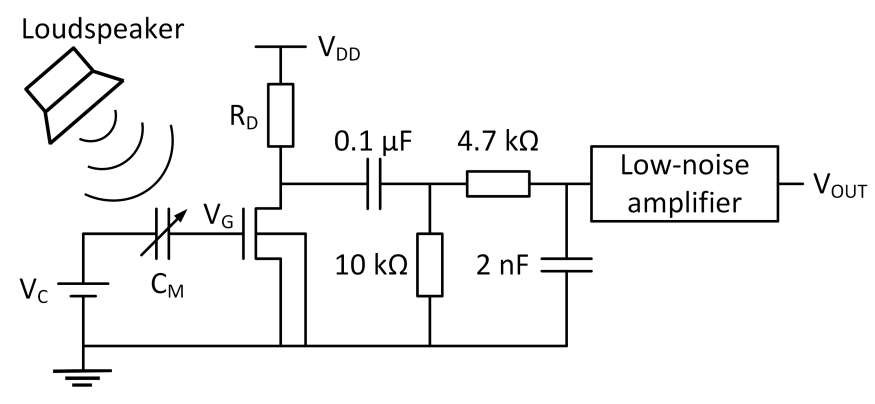

Fig. 6. Schematic of measurement circuit. A loudspeaker generates sound pressure that is directed toward the graphene-based membrane. The variable capacitor, $C_{M}$, formed by the membrane and the bottom electrode is connected to a bias voltage, $V_{C}$, and to the gate of a MOSFET. The drain of the MOSFET is connected to a passive band-pass filter, the output of which is connected to a low-noise amplifier (Zoom F4 Multi-Track Field Recorder).

where $x_{\text {peak }}$ is the maximum deflection of the membrane during oscillation. (7) is then substituted into (6), resulting in

$$
V_{G}=V_{C}-\frac{Q_{0} d}{\varepsilon A}+\frac{Q_{0} x_{p e a k}}{\varepsilon A} \sin \left(\omega_{S P} t\right)
$$

which describes the response of $V_{G}$ as a function of time. A value of $380 \Omega$ has been used for the drain resistor, $R_{D}$, and $V_{D D}$ has been set to $13.05 \mathrm{~V} . V_{C}$ has been fixed at $1 \mathrm{~V}$, which results in a $V_{G}$ of $0.8 \mathrm{~V}$, turning on the MOSFET with $V_{D}=9.18 \mathrm{~V}$. There will be a resulting voltage drop of $0.2 \mathrm{~V}$ across $C_{M}$. As the membrane vibrates in response to a sound pressure, the value of $C_{M}$ will vary, which will in turn vary the voltage drop across $C_{M}$. The value of $V_{G}$ will vary in response and the resulting change in the drain current will give an AC signal on top of the DC value of $V_{D}$.

The drain terminal of the MOSFET has been connected to a passive band-pass filter with the component values specified in Fig. 6. The lower and upper cut-off frequencies of the filter are $159 \mathrm{~Hz}$ and $16.8 \mathrm{kHz}$, respectively, so that electrical noise above and below the audio band are eliminated from the output signal. The output from the readout circuit has been connected to an low-noise audio interface (Zoom F4 Multi-Track Field Recorder) that amplifies the signal by $20 \mathrm{~dB}$, giving an output voltage, $V_{O U T}$.

In order to characterize the response of the device to sound pressure at frequencies across the audio band, the microphone chip has been secured in an audio chamber along with the readout circuit. As shown in Fig. 7, a loudspeaker has been mounted to the top of a funnel that directs sound from the loudspeaker to the audio chamber, which is equipped with electrical feedthroughs, allowing for the necessary connections to be made to the device and readout circuitry. A signal generator producing an AC sinusoidal signal has been connected to the loudspeaker so that a sound pressure is produced. The resulting output signal from the readout circuit is amplified by the audio interface, the output of which $\left(V_{O U T}\right)$ has been connected to a spectrum analyzer. The performance of the device has been characterized for different frequencies across the audio range and for each frequency, averaging has been performed to eliminate noise and allow for the amplitude of the



Fig. 7. Audio experimental set-up. Device has been placed in the audio chamber directly below the funnel that directs sound pressure generated by the loudspeaker.

output voltage signal to be measured. The chamber does not have a temperature control feature so all measurements have been performed at room temperature. Varying the temperature could affect device performance, influencing the stiffness of the membrane as the different materials expand and contract, so a possible future area of study could be characterizing device performance at different temperatures.

\section{Measurement Results and Discussion}

The experimental set-up that has been detailed in the previous section has been used to characterize the response to sound pressure of the graphene-based membrane microphone device shown in Fig. 2. The loudspeaker has been set to produce an RMS sound pressure of $0.2 \mathrm{~Pa}\left(80 \mathrm{~dB}_{\mathrm{SPL}}\right)$. The sound pressure level has been measured with a sound level meter (Cirrus Research Optimus Red CR:162C) positioned at the bottom of the funnel, to validate the sound pressure that the device is subjected to. At the same time as the loudspeaker is producing a sound pressure, the output RMS voltage from the audio

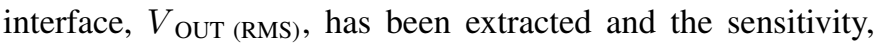
$S_{\mathrm{P}}$, in $\mathrm{mV} / \mathrm{Pa}$, to sound pressure has been calculated as

$$
S_{p}=\frac{V_{\mathrm{OUT}(\mathrm{RMS})}}{\bar{p}}
$$

where $\bar{p}$ is the RMS sound pressure level in Pa. Also, the sensitivity value relative to $1 \mathrm{~V} / \mathrm{Pa}$ can be expressed in $\mathrm{dBV}$. The measurement has been performed across the frequency range of $100 \mathrm{~Hz}$ to $20 \mathrm{kHz}$ in increments of $100 \mathrm{~Hz}$ up to 
TABLE I

COMPARISON OF SENSITIVITY VALUES

\begin{tabular}{|c|c|c|}
\hline Membrane Type & Reference & Sensitivity (dBV) \\
\hline Graphene & {$[13]$} & -70 \\
Graphene-PMMA & This work & -40 \\
Graphene-PMMA & {$[14]$} & -20 \\
Silicon & {$[11]$} & -28 \\
\hline
\end{tabular}

$1 \mathrm{kHz}$ and in increments of $1 \mathrm{kHz}$ between $1 \mathrm{kHz}$ and $10 \mathrm{kHz}$, with the sound pressure being maintained at $0.2 \mathrm{~Pa}\left(80 \mathrm{~dB}_{\mathrm{SPL}}\right)$. Then, the entire series of measurements across the frequency range has been repeated for sound pressure values of $0.112 \mathrm{~Pa}$ $\left(75 \mathrm{~dB}_{\mathrm{SPL}}\right)$ and $0.063 \mathrm{~Pa}\left(70 \mathrm{~dB}_{\mathrm{SPL}}\right)$. Fig. 8 shows the extracted sensitivity values plotted against the audio frequency for all three sound pressures.

It can be seen that the system exhibits a sound pressure sensitivity between $0.1 \mathrm{mV} / \mathrm{Pa}$ and $10 \mathrm{mV} / \mathrm{Pa}$, or between $-80 \mathrm{dBV}$ and $-40 \mathrm{dBV}$, across the frequency range from $100 \mathrm{~Hz}$ to $20 \mathrm{kHz}$. It can be seen from Fig. 8 that the frequency response is consistent, not varying by more than $0.2 \mathrm{mV} / \mathrm{Pa}$, for the three different sound pressures that have been used. For audio frequencies of $6 \mathrm{kHz}$ and higher, the minimum sound pressure that has been detected successfully using the experimental set-up is $0.063 \mathrm{~Pa}\left(70 \mathrm{~dB}_{\mathrm{SPL}}\right)$. For frequencies across the range $100 \mathrm{~Hz}$ to $20 \mathrm{kHz}$, sound pressures down to $0.112 \mathrm{~Pa}\left(75 \mathrm{~dB}_{\mathrm{SPL}}\right)$ have been detected successfully.

The values of sensitivity for graphene-based membrane microphone devices previously reported have ranged from $-70 \mathrm{dBV}$ up to $-20 \mathrm{dBV}$ [13], [14]. In addition to possessing comparable sensitivity to other work, the device reported in this paper exhibits sensitivity that is fairly constant, remaining between $0.1 \mathrm{mV} / \mathrm{Pa}$ and $10 \mathrm{mV} / \mathrm{Pa}$, from $100 \mathrm{~Hz}$ up to $20 \mathrm{kHz}$. Also, this work reports a more constant sensitivity for different sound pressures compared to previous work [14]. In addition, the device in this work has been shown to operate with significantly lower DC bias voltages, about $1 \mathrm{~V}$, applied to the membrane and fixed electrode.

\section{CONCLUSION}

In this paper, the design, fabrication and characterization of a graphene-based membrane operating as a MEMS microphone have been reported. The device has been fabricated using a novel process, featuring a graphene/PMMA bilayer membrane that has been transferred over a cavity on a separate chip before being affixed to the surface of another chip containing an electrode. The process resulted in the successful fabrication of a $7 \mathrm{~mm} \times 7 \mathrm{~mm} \times 0.78 \mathrm{~mm}$ device with a membrane-to-electrode gap of $8.8 \mu \mathrm{m}$, allowing for the device to operate with low DC bias voltages of about $1 \mathrm{~V}$. The measured sensitivity across the frequency range $100 \mathrm{~Hz}$ up to $20 \mathrm{kHz}$ is fairly constant, remaining between $0.1 \mathrm{mV} / \mathrm{Pa}$ and $10 \mathrm{mV} / \mathrm{Pa}$. Lastly, the values of sensitivity do not vary by more than $0.2 \mathrm{mV} / \mathrm{Pa}$ when the sound pressure is increased from $70 \mathrm{~dB}_{\mathrm{SPL}}$ to $80 \mathrm{~dB}_{\mathrm{SPL}}$.

\section{REFERENCES}

[1] K. S. Novoselov, A. K. Geim, S. V. Morozov, D. Jiang, Y. Zhang, S. V. Dubonos, I. V. Grigorieva, and A. A. Firsov, "Electric field effect in atomically thin carbon films," Science, vol. 306, no. 5696, pp. 666-669, Oct. 2004.

[2] C. Lee, X. D. Wei, J. W. Kysar, and J. Hone, "Measurement of the elastic properties and intrinsic strength of monolayer graphene," Science, vol. 321, no. 5887, pp. 385-388, July 2008.

[3] J. Aguilera-Servin, T. Miao, and M. Bockrath, "Nanoscale pressure sensors realized from suspended graphene membrane devices," Appl. Phys. Lett., vol. 106, no. 8, p. 083103, Feb. 2015.

[4] M. Pumera, "Graphene in biosensing," Mater. Today, vol. 14, no. 7-8, pp. 308-315, July-Aug. 2011.

[5] M. H. Zarei and M. J. Sharifi, "Graphene nanoribbon photodetectors based on an asymmetric potential barrier: a new concept and a new structure," J. Comput. Electron., vol. 17, pp. 531-539, Feb. 2018.

[6] M. Ahmadian and M. J. Sharifi, "A diagrammatic approach to singleelectron spintronics and a new analytical model for ferromagnetic singleelectron transistors," Int. J. Electron. Commun., vol. 102, pp. 62-68, Feb. 2019.

[7] M. Ahmadian and K. Jafari, "A Graphene-Based Wide-Band MEMS Accelerometer Sensor Dependent on Wavelength Modulation," IEEE Sens. J., Apr. 2019.

[8] Q. G. Wang, Y. F. Wang, and L. Dong, "MEMS Flow Sensor Using Suspended Graphene Diaphragm With Microhole Arrays," J. Microelectromech. Syst., vol. 27, no. 6, pp. 951-953, Dec. 2018.

[9] Y. Lee, S. Bae, H. Jang, S. Jang, S. E. Zhu, S. H. Sim, Y. I. Song, B. H. Hong, and J. H. Ahn, "Wafer-scale synthesis and transfer of graphene films," Nano Letters, vol. 10, no. 2, pp. 490-493, Feb. 2010.

[10] R. N. Miles, W. L. Cui, Q. T. Su, and D. Homentcovschi, "A MEMS Low-Noise Sound Pressure Gradient Microphone With Capacitive Sensing," J. Microelectromech. Syst., vol. 24, no. 1, pp. 241-248, Feb. 2015.

[11] E. Graf, W. Kronast, S. Duhring, B. Muller, and A. Stoffel, "Silicon membrane condenser microphone with integrated field-effect transistor," Sens. Actuators A, Phys., vol. 37-38, pp. 708-711, July-Aug. 1993.

[12] Y. Kusano, J. Segovia-Fernandez, S. Sonmezoglu, R. Amirtharajah, and D. A. Horsley, "Frequency selective mems microphone based on a bioinspired spiral-shaped acoustic resonator," in 19th Int. Conf. SolidState Sens., Actuators and Microsyst. (Transducers), June 2017, pp. 7174

[13] D. Todorovic, A. Matkovic, M. Milicevic, D. Jovanovic, R. Gajic, I. Salom, and M. Spasenovic, "Multilayer graphene condenser microphone," 2 M Mater., vol. 2, no. 4, Dec. 2015.

[14] S. Woo, J. H. Han, J. H. Lee, S. Cho, K. W. Seong, M. Choi, and J. H. Cho, "Realization of a high sensitivity microphone for a hearing aid using a graphene-pmma laminated diaphragm," ACS Appl. Mater. Interfaces, vol. 9, no. 2, pp. 1237-1246, Jan. 2017.

[15] J. S. Bunch, A. M. van der Zande, S. S. Verbridge, I. W. Frank, D. M. Tanenbaum, J. M. Parpia, H. G. Craighead, and P. L. McEuen, "Electromechanical resonators from graphene sheets," Science, vol. 315, no. 5811, pp. 490-493, Jan. 2007.

[16] S. P. Koenig, N. G. Boddeti, M. L. Dunn, and J. S. Bunch, "Ultrastrong adhesion of graphene membranes," Nature Nanotechnol., vol. 6, no. 9, pp. 543-546, Sep. 2011.

[17] J. Sun, M. E. Schmidt, M. Muruganathan, H. M. H. Chong, and H. Mizuta, "Large-scale nanoelectromechanical switches based on directly deposited nanocrystalline graphene on insulating substrates," Nanoscale, vol. 8, no. 12, pp. 6659-6665, Mar. 2016.

[18] E. Grady, E. Mastropaolo, T. Chen, A. Bunting, and R. Cheung, "Low frequency graphene resonators for acoustic sensing," Microelectron. Eng., vol. 119, pp. 105-108, May 2014.

[19] A. K. Al-mashaal, G. S. Wood, A. Torin, E. Mastropaolo, M. J. Newton, and R. Cheung, "Dynamic behavior of ultra large graphenebased membrances using electrothermal transduction," Appl. Phys. Lett., vol. 111, no. 24, p. 243503, Dec. 2017.

[20] A. K. Al-mashaal, G. S. Wood, A. Torin, E. Mastropaolo, M. J Newton, and R. Cheung, "Tunable graphene-polymer resonators for audio frequency sensing applications," IEEE Sens. J., Nov. 2018.

[21] Cirrus Logic Data Sheet - CS7331P. [Online]. Available: https://www.cirrus.com/products/cs7331/

[22] V. Kaajakari, Practical MEMS. Small Gear Publishing, 2009.

[23] S. D. Senturia, Microsystem Design. Springer, 2001.

[24] S. Lai, P. Cosseddu, A. Bonfiglio, and M. Barbaro, "Ultralow voltage pressure sensors based on organic FETs and compressible capacitors," Electron Device Lett., vol. 34, no. 6, pp. 801-803, June 2013. 


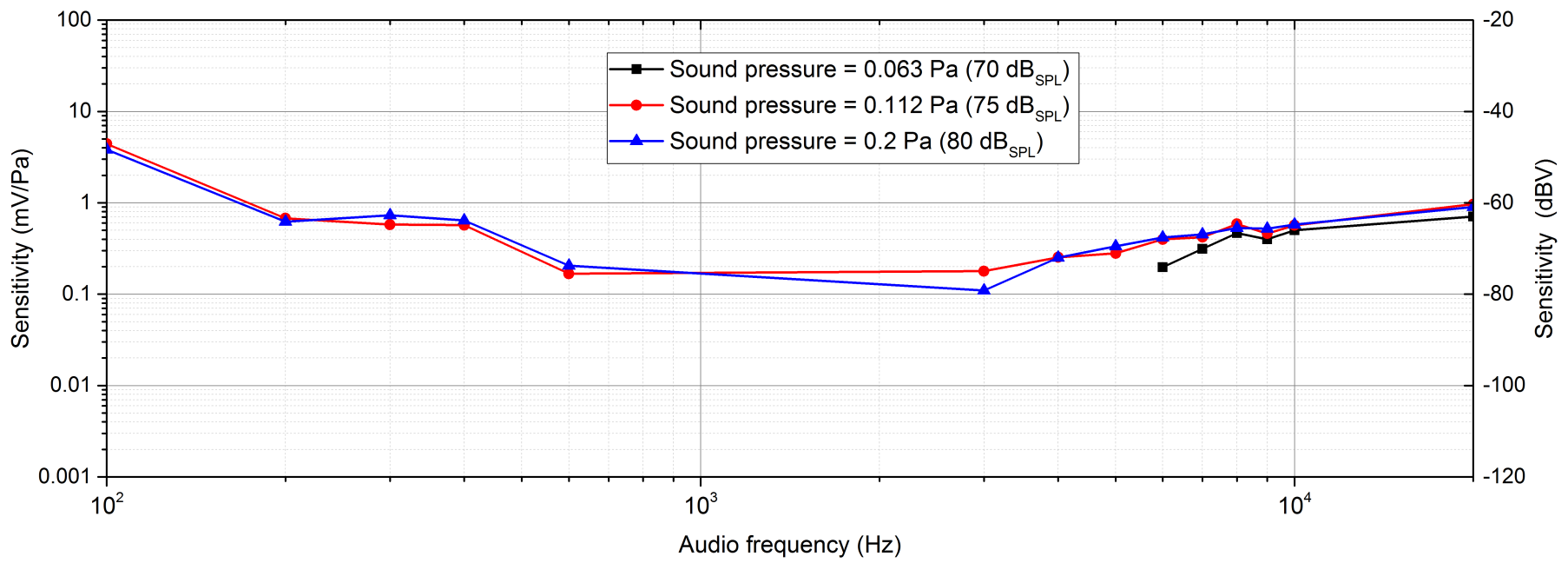

Fig. 8. Response of the graphene-based membrane MEMS microphone system sensitivity as a function of audio frequency.

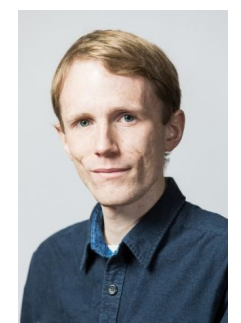

Graham S. Wood (M'10) received the M.Eng degree in electronics and electrical engineering and the M.Sc. degree in microelectronics from the University of Edinburgh, Edinburgh, U.K., in 2008 and 2011 , respectively, and the Ph.D. degree in microelectromechanical systems from the University of Southampton, Southampton, U.K., in 2016.

$\mathrm{He}$ is currently a Research Associate with the School of Engineering, Institute for Integrated Micro and Nano Systems, University of Edinburgh. He held the same position from 2008 to 2010 , where he conducted research concerning the actuation and sensing of SiC MEMS resonators for high frequency RF applications. His current research involves the use of graphene resonating structures as acoustic transducers and the use of nanocomposite materials in flexible force sensors.

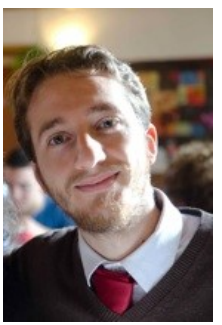

Alberto Torin His doctoral thesis focused on the numerical modeling of percussion instruments. $\mathrm{He}$ remained with the Acoustics and Audio Group at the University of Edinburgh for a further two years as a Postdoctoral Research Associate, working on numerical modeling and experimental validation of a novel graphene-based MEMS microphone. He is currently a freelance technical writer with a penchant for programming and data science.

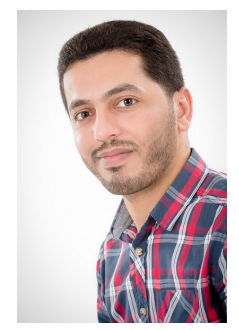

Asaad K. Al-mashaal received the B.Sc. degree in physics and the M.Sc. degree in molecular quantum mechanics, physics from Basrah University, Basrah, Iraq, in 2007 and 2009, respectively.

$\mathrm{He}$ is currently pursuing the $\mathrm{Ph} . \mathrm{D}$. degree in electronics and electrical engineering with the School of Engineering, Institute for Integrated Micro and Nano Systems, University of Edinburgh, Edinburgh, U.K. His research interests include design, simulation, and fabrication of graphene-based resonators/actuators and MEMS/NEMS sensors for audio applications.

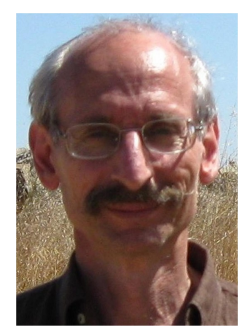

Leslie S. Smith Having worked on parallel systems, he moved on to working on neural networks, gradually exploring both real (biological) and artificial (neuromorphic) implementations. He became interested in the auditory area initially as a source of time-varying data, and remained interested after discovering the problems posed by this area. $\mathrm{He}$ is currently a Professor with Computing Science and Mathematics, University of Stirling, Stirling, U.K, and is currently researching cochlea-like transducers as well as neuroinformatics.

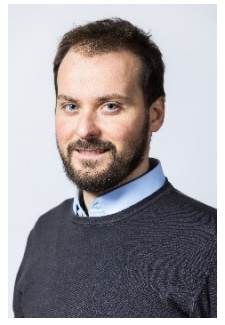

Enrico Mastropaolo received the Laurea degree in micro-electronics engineering from the Univeristà degli Studi di Padova, Padova, Italy, in 2006, and the $\mathrm{Ph} . \mathrm{D}$. degree in microsystems and microfabrication from the University of Edinburgh, Edinburgh, U.K., in 2011

$\mathrm{He}$ is currently a Lecturer in mechanical engineering in the School of Engineering, Institute for Integrated Micro and Nano Systems, University of Edinburgh. His research focuses on microelectromechanical systems (MEMS), their design, simulation, fabrication and characterization with special interest in transduction techniques and the dynamics of microstructures. Currently, he is working on polymerbased structures, embedment of polymer nanocomposites and nanostructures in microsystems, interaction of micro and small-scale structures with fluid flows.

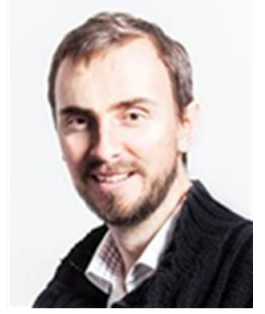

Michael J. Newton received the B.Sc. degree in physics and the Ph.D degree in physics with a specialization in acoustics from the University of Edinburgh, Edinburgh, U.K., in 2004 and 2009, respectively.

From 2010 until early 2012 he was with Computing Science and Mathematics, University of Stirling, Stirling, U.K., where he worked on the development of novel microphone technology and auditory modeling. He is currently a Lecturer in acoustics and audio signal processing in the Acoustics and Audio Group at the University of Edinburgh. His research interests span microsystem design for audio sensing, spatial audio, and musical acoustics. 


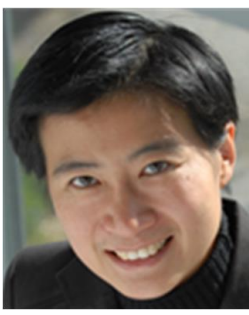

Rebecca Cheung (M'96-SM'02) received first class honors and the Ph.D. degrees in electronics and electrical engineering from the University of Glasgow, Glasgow, U.K., in 1986 and 1990, respectively.

During her Ph.D. work, she was a Visiting Researcher at the IBM Thomas J. Watson Research Center, Yorktown Heights, NY, where high-density plasma-etching techniques were developed to form nanostructured GaAs. From 1990 to 2000, she was a Visiting Scientist at the Delft Institute of Microelectronics and Submicron Technology, Delft, The Netherlands; the Laboratory for Electromagnetic Fields and Microwave Electronics, Eidgenössische Technische Hochschule Zürich, Zürich, Switzerland; and the Nanoelectronics Research Centre, University of Glasgow, working on various topics related to semiconductor technology, process induced material damage, mesoscopic physics in SiGe heterostructures, and microwave circuits in InP for gigabit electronics. Additionally, she was a Founding Member of the Nanostructure Engineering Science and Technology Group, University of Canterbury, Christchurch, New Zealand, in 1998. She currently holds a Chair in Nanoelectronics in the School of Engineering, University of Edinburgh, Edinburgh, U.K.

Dr. Cheung has an international reputation for her contribution in the development and application of micro- and nano- fabrication. More recently, her research focuses on microresonators and microelectromechanical systems. She has published over 200 scientific articles, 1 patent and 1 book in related topics. 